\title{
Chemodynamical Evolution of Elliptical Galaxies: Metallicity Gradients and Scaling Relations
}

\author{
Chiaki Kobayashi ${ }^{1,2}$ \\ ${ }^{1}$ Max Planck Institute for Astrophysics, 85741 Garching, Germany \\ ${ }^{2}$ E-mail: chiaki@mpa-garching.mpg.de
}

Received 2004 January 27, accepted 2004 March 22

\begin{abstract}
We simulate the chemodynamical evolution of a hundred elliptical galaxies using our GRAPE-SPH code, and succeed in reproducing the radial metallicity gradients and the global scaling relations such as the fundamental plane. These observations cannot be explained by either monolithic collapse or by major merger alone. Rather it requires a model in which both formation processes arise, such as the present CDM scheme.
\end{abstract}

Keywords: methods: $N$-body simulations — galaxies: abundances — galaxies: elliptical and lenticular, cD - galaxies: evolution — galaxies: formation

\section{Introduction}

For the formation of elliptical galaxies, two competing scenarios have so far been proposed: elliptical galaxies should form monolithically by gravitational collapse of gas clouds with considerable energy dissipation (e.g. Larson 1974; Arimoto \& Yoshii 1987), or alternatively ellipticals should form through mergers of gaseous disk galaxies or of many dwarf galaxies (e.g. Kauffmann et al. 1993; Baugh et al. 1996).

Most ellipticals have radial metallicity gradients and the metallicity gradients do not correlate with mass or metallicity (Kobayashi \& Arimoto 1999). If elliptical galaxies form monolithically from a massive gas cloud, the metallicity gradient should correlate with the global properties of galaxies in the sense that more massive galaxies have steeper gradients (Carlberg 1984). This discrepancy could be solved if mergers flatten the original gradient. Indeed, numerical simulations showed that the gradient in a disk galaxy should be halved after three successive mergers of galaxies with similar size (White 1980). However, simulations of both dissipative collapse and mergers leave room for improvements, because essential physical processes such as star formation, feedback of supernovae, and metal enrichment were not taken into account.

All giant elliptical galaxies follow tight correlations among global properties, such as the colourmagnitude relation, the metallicity-velocity dispersion relation, and the fundamental plane. This can be explained by the assumption that the bulk of stars in ellipticals form during an initial starburst at high redshift and that the star formation is terminated by a supernovaedriven galactic wind that expels the left-over interstellar gas from galaxies. However, there exists an intrinsic scatter along the fundamental plane and its origin is unknown.
Here we simulate the chemodynamical evolution of elliptical galaxies based on the CDM picture. In the CDM cosmology, galaxies should form through the successive merging of subgalaxies with various masses. In contrast with the semi-analytic models, we exclude the assumption that elliptical galaxies form only from the major merger of disk galaxies. Instead we allow various merging histories for elliptical galaxies. In some cases, an elliptical galaxy forms by an assembly of gas-rich small galaxies, which looks like a monolithic collapse. In other cases, the evolved galaxies with little gas merge to form an elliptical galaxy. By reproducing the metallicity gradients and the scaling relations, we discuss the origin of elliptical galaxies.

\section{The Model}

We simulate the formation and chemodynamical evolution of galaxies with the GRAPE-SPH chemodynamical model that includes various physical processes associated with the formation of stellar systems: radiative cooling, star formation, feedback of Type II and Ia supernovae and of stellar winds, and chemical enrichment (see Kobayashi 2004 for the detail, hereafter K04). For the feedback of energy and heavy elements, we exclude the instantaneous recycling approximation. For $\mathrm{SNe}$ Ia, we adopt the single degenerate scenario with the metallicity effect (Kobayashi et al. 1998, 2000). The photometric evolution is calculated with the spectral synthesis population database taken from Kodama \& Arimoto (1997).

As the initial condition, we adopt $1 \sigma$ or $3 \sigma$ over dense regions of the CDM initial fluctuation with $z \sim 25$, a co-moving radius of $\sim 1.5 \mathrm{Mpc}$, a mass of $\sim 10^{12} \mathrm{M}_{\odot}$ (baryon fraction of 0.1 ), $\sim 10000$ and 60000 particles (half for gas and the rest for dark matter), and a spin parameter $\lambda \sim 0.02$. Throughout the paper, we set the cosmological parameters of $H_{0}=50 \mathrm{~km} \mathrm{~s}^{-1} \mathrm{Mpc}^{-1}, \Omega_{\mathrm{m}}=1.0$, $\Omega_{\Lambda}=0$, and $\sigma_{8}=1.0$. 


\section{Merging Histories}

We simulate 72 fields with different cosmological initial conditions, and obtain 124 galaxies (78 ellipticals and 46 dwarfs). Different galaxies undergo different evolution histories. The difference is seeded in the initial condition. Galaxies form through the successive merging of subgalaxies with various masses, which varies between a major merger at one extreme and a monolithic collapse of a slowly rotating gas cloud at the other. We classify galaxies into the following five classes according to their merging histories.

(E1) Monolithic: Galaxies form through the assembly of many ( $\gtrsim 10)$ gas-rich subgalaxies with stellar masses of $M \sim 10^{9} \mathrm{M}_{\odot}$. Such assembly has generally finished by $z \sim 3$, and at least by $z \sim 2$. The material of subgalaxies quietly accretes onto the central galaxy. It is difficult to discriminate these subgalaxies, and this assembly looks like a monolithic collapse.

(E2) Assembly: Galaxies form through the assembly of subgalaxies with $M \sim 10^{10} \mathrm{M}_{\odot}$. Each subgalaxy has an evolved core, which merges violently with the others. While the subgalaxy passes through the central galaxy many times, many stars of the galaxy are stripped and some of them accrete on again.

(E3) Minor merger: The formation of the main component of the present-day galaxy is the same as class (E1) or (E2), but these galaxies undergo minor merger events at $z \lesssim 3$. We define a minor merger as when the stellar mass ratio of the merging galaxies, $f \equiv M_{2} / M_{1}$, ranges from $\sim 0.01$ to $\sim 0.2$. With such minor merger events, the surface-brightness profile and the metallicity gradients are not affected so much.

(E4) Major merger: Galaxies undergo a major merger with $f \gtrsim 0.2$ at $z \lesssim 3$. The redshift of $z \sim 3$ generally corresponds to the galaxy formation epoch and the major merger occurs after most stars in the presentday galaxies form. The merger event destroys the metallicity gradient that has existed in the premerger galaxy in a way depending on the mass ratio, $f$, and the gas mass of the secondary galaxy (see K04).

(E5) Multiple major mergers: Galaxies undergo a major merger $(f \gtrsim 0.2)$ at $z \lesssim 3$ and one or two other mergers with $f \gtrsim 0.1$ at later time.

Dwarf galaxies with $M_{\mathrm{V} \text {,tot }} \gtrsim-19 \mathrm{mag}$ are classified into the following four classes, according to their starformation histories. Observationally, the first class of galaxies is dwarf ellipticals, the others are dwarf irregulars.

(D1) Initial starburst: Galaxies form with the initial starburst at $z \gtrsim 1$. In some galaxies, many supernova explosions occur and cause galactic winds. In other galaxies, the gas is not ejected completely, but the gas density is so small that only few stars form at lower redshifts. Thus, the colours are red and these dwarfs follow the same colour-magnitude relation as giant ellipticals.
(D2) Continuous star formation: Galaxies grow though continuous star formation. After the initial starburst, there are the accretion of gas clumps and/or the interaction with other galaxies, which make the star formation continue to lower redshifts.

(D3) Continuous starformation with recent starburst: The same as D2, but a starburst occurs at the recent 2-3 Gyr. Thus, the galaxy colours are blue.

(D4) Recent starburst: Galaxies form through recent starbursts at $z \sim 0.7$. Such starbursts are induced by gas accretion and/or galaxy interactions.

The numbers and percentages of galaxies in each class are (E1) 5 [4.0\%], (E2) 18 [15\%], (E3) 19 [15\%], (E4) 25 [20\%], (E5) 11 [8.9\%], (D1) 20 [16\%], (D2) 13 [10\%], (D3) 9 [7.3\%], and (D4) 4 [3.2\%]. The percentages of non-major-merger [(E1)-(E3)] and major-merger galaxies [(E4), (E5)] are 34\% and 29\%, respectively. Although we show the results both for giant and dwarf galaxies in the following figures, the resolution of the dwarf galaxies is not enough because the particle number in a galaxy is small ( $\sim 200$ in the worst case) and the gravitational softening ( $1.0 \mathrm{kpc}$ for the low resolution) is comparable to the size of galaxies.

\section{Metallicity Gradients}

All galaxies have the de Vaucouleurs' surface brightness profile and we derive the effective radius, $r_{\mathrm{e}}$, by fitting Vaucouleurs' law. A $V$-band luminosity-weighted metallicity is calculated at a projected radius, and a metallicity gradient is estimated as a radial variation of the metallicity in $r \leq 2 r_{\mathrm{e}}$. Figure 1 shows that there is no correlation between metallicity gradients and stellar masses (in $r_{\mathrm{e}}$ ): (a) The average oxygen gradient is $\sim-0.3$ and the dispersion is \pm 0.2 , which are both consistent with observations of $\mathrm{Mg}_{2}$ gradients (Kobayashi \& Arimoto 1999); (b) For iron, the average is $\sim-0.4$, which is steeper than oxygen. This is because the late star formation increases the iron abundance at the center.

The origin of scatter is clearly shown with the symbols: the galaxies that form monolithically (filled circles and squares) have steeper gradients and the galaxies that undergo major mergers (open circles and squares) have shallower gradients. Therefore, we conclude that the metallicity gradients do not depend on the galaxy mass and the variety of the gradients stems from the difference in the merging history.

Figure 2 shows the histograms of the metallicity gradients for the non-major-merger galaxy [(E1)-(E3), grey area] and the major-merger galaxy [(E4), (E5), hatched area] compared with the observation (thick line). The distributions for non-major-merger and major-merger galaxies are different, with peaks at $\Delta \log Z / \Delta \log r \sim-0.3$ and -0.2 , respectively. The observed variation in the metallicity gradients cannot be explained by either the monolithic collapse only, nor the major merger only. It is well reproduced in the present model where both formation processes arise under the CDM scheme. 


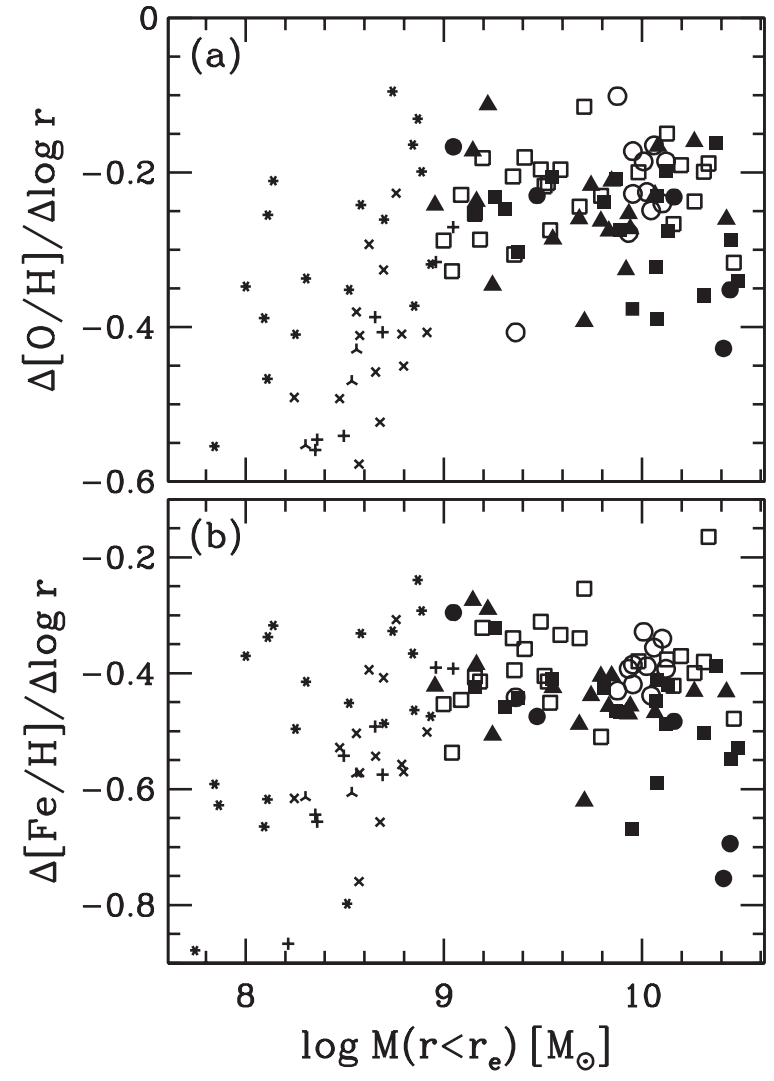

Figure 1 No correlation between metallicity gradients and stellar masses in $r_{\mathrm{e}}$. The symbols that show the merging histories for elliptical galaxies and star formation histories for dwarf galaxies are (E1) monolithic [filled circles], (E2) assembly [filled squares], (E3) minor merger [filled triangles], (E4) major merger [open squares], (E5) multiple major merger [open circles], (D1) initial starburst [asterisks], (D2) continuous star formation [crosses], (D3) continuous star formation with recent star burst [plus], and (D4) recent starburst [three-pointed stars].

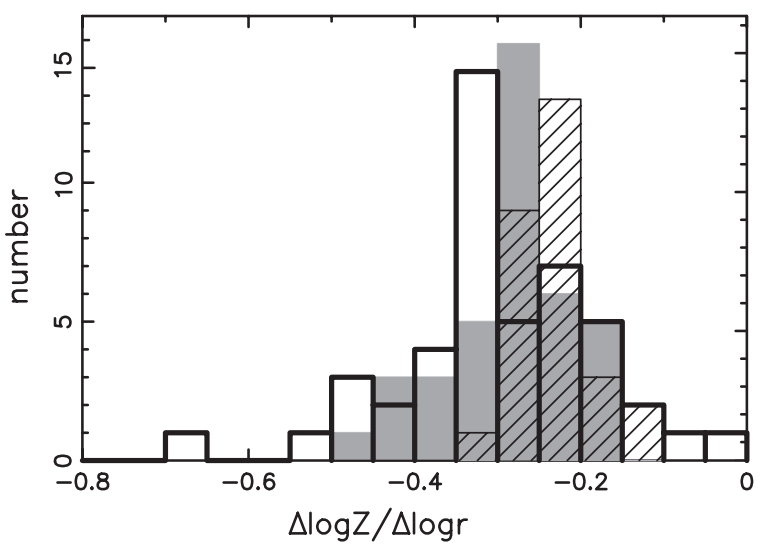

Figure 2 The histograms of the metallicity gradients for non-major-merger (gray area) and major-merger galaxies (hatched area). The thick lines show the observation with a $\mathrm{Mg}_{2}$ index from Kobayashi \& Arimoto (1999).

\section{Scaling Relations}

The variety of the merging histories in the CDM picture produces the observed variety of the internal structure of elliptical galaxies. On the other hand, the observed elliptical galaxies follow tight correlations among global properties such as the colour-magnitude relation, the metallicity-velocity dispersion relation, and the fundamental plane. As discussed in K04, the galaxies of the K04 simulation are more extended and, thus, there is an offset in the radius-magnitude relation. This is because the star formation takes place too early, before the gas accretes towards the centre, and can be solved by changing the star formation timescale, i.e. reducing the star formation parameter $c\left(t_{\mathrm{sf}}=t_{\mathrm{dyn}} / c\right)$.

Here we show the results with $c=0.1$, instead of 1.0 used in Section 4, using the same initial conditions as in Section 4. The calculation is done using the GRAPE5 system in the National Astronomical Observatory of Japan during 2003. The metallicity increases because of the longer timescale of star formation, but the gradient dose not change as much (see Figure 14 in K04). In addition, we add a new sample of $\mathrm{cD}$ galaxies using wider initial conditions with the radius $\sim 3 \mathrm{Mpc}$ and $N \sim 60000$. There is a gap in the mass/luminosity between this sample and the K04 sample. As shown in Figure 3, we succeed in reproducing the following global scaling relations from cD galaxies to dwarf ellipticals.

(a) Faber-Jackson: The simulated giant galaxies follow the observed relation (the gray points) of $L \propto \sigma^{n}$ with $n=4$ (solid line). The dispersion is very small and a little smaller than the observation. For smaller galaxies with $M_{\mathrm{V} \text {,tot }} \gtrsim-20 \mathrm{mag}$, the relation of the simulated galaxies looks to have a steeper slope $(n \sim 3.5)$. However, as noted in Section 3, the resolution of the simulated dwarf galaxies is not enough.

(b) Luminosity-effective radius: Massive ellipticals have larger effective radii. The dispersion is not small, but is comparable to the observation $(\sim 0.5 \mathrm{mag})$. In the simulation, the surface brightness of the central part is smeared by the gravitational softening, which causes an uncertainty in the de Vaucouleurs' fit. For dwarf ellipticals, the observed relation has a shallower slope than that of giant ellipticals. This tendency can be seen in the simulated dwarfs although the scatter is larger.

(c) Surface brightness-effective radius: The simulated giant ellipticals follow the observed relation where larger galaxies have lower surface brightness in $r_{\mathrm{e}}$. The scatter is almost the same as observed. For dwarf galaxies, the observed relation is rectangular, and larger galaxies have higher surface brightnesses. The simulated dwarf galaxies are populated at the same side of the observed relation, but the direction of the relation is different from the observation. The effective radii of the simulated dwarfs tend to be too large, which is due to the lack of resolution.

Figure 4 shows the fundamental plane in $\kappa$-space (Bender et al. 1992). The parameters $\kappa_{1}, \kappa_{2}$, and $\kappa_{3}$ express mass, surface brightness, and mass-to-light ratio, 

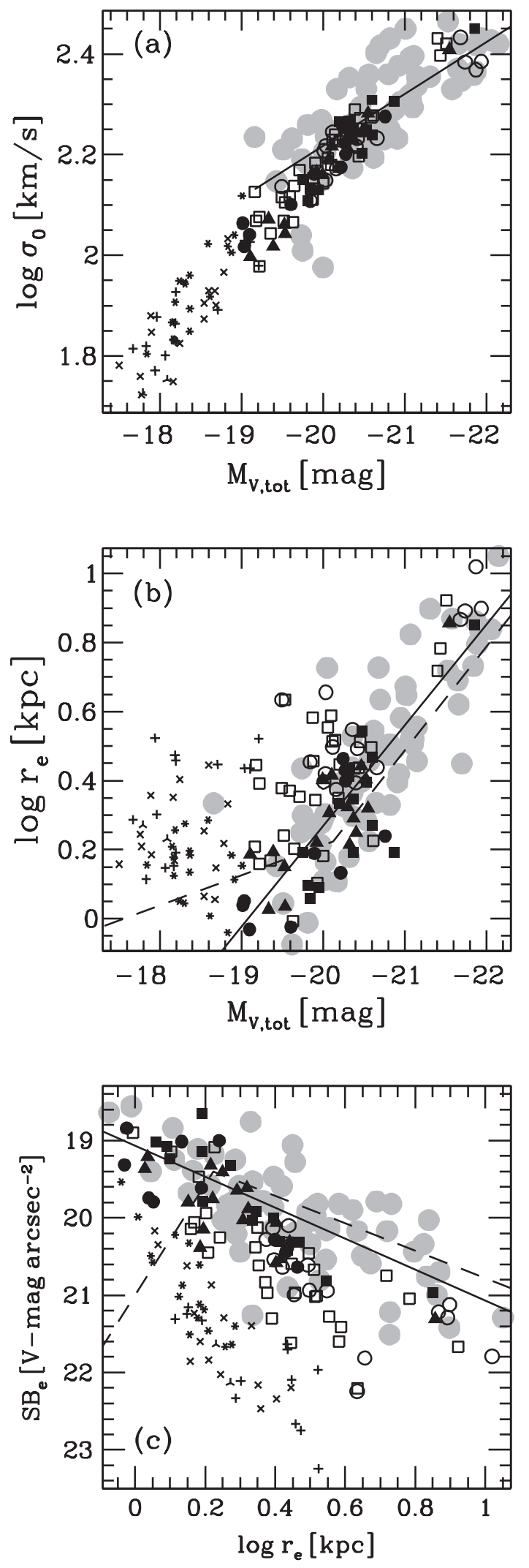

Figure 3 The Faber-Jackson relation (a), the luminosity-effective radius relation (b), and the surface brightness-effective radius relation (c). The symbols are the same as in Figure 1. The solid and dashed lines respectively show the observed relations of giant galaxies of Coma cluster (Pahre 1999, also shown with the gray points) and dwarf galaxies of Virgo cluster (Binggeli et al. 1984).

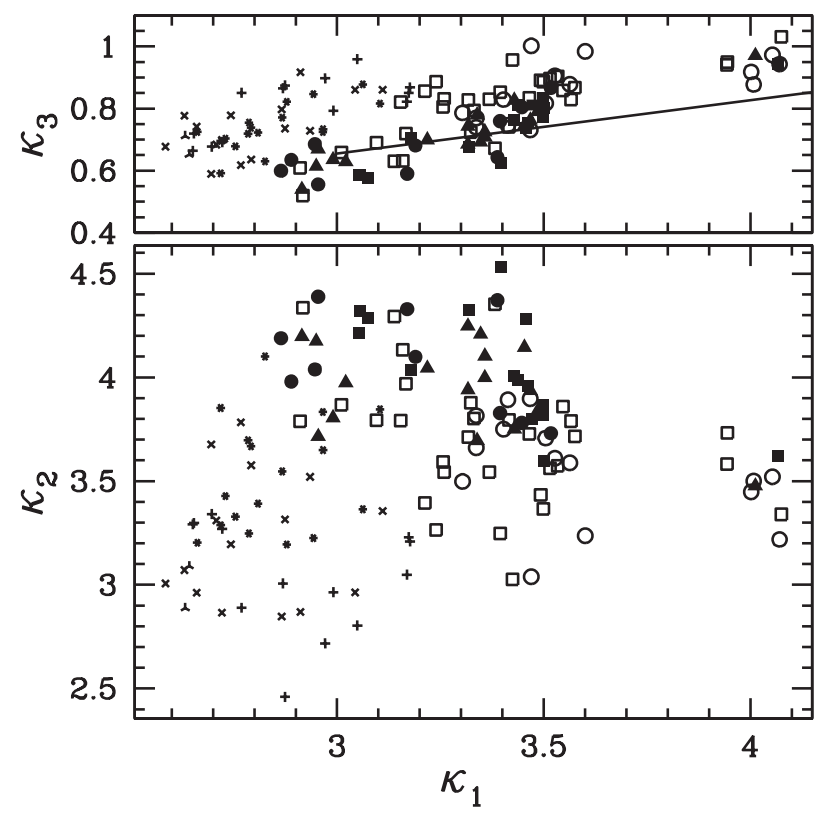

Figure 4 The fundamental plane shown in $\kappa$-space; the edge-on view (upper panel) and the face-on view (lower panel). The symbols are the same as in Figure 1. The solid line shows the observed relation (Pahre 1999).

respectively, and are defined as

$$
\begin{aligned}
& \kappa_{1} \equiv\left(2 \log \sigma_{0}+\log r_{\mathrm{e}}\right) / \sqrt{2}, \\
& \kappa_{2} \equiv\left(2 \log \sigma_{0}+2 \log I_{\mathrm{e}}-\log r_{\mathrm{e}}\right) / \sqrt{6}, \\
& \kappa_{3} \equiv\left(2 \log \sigma_{0}-\log I_{\mathrm{e}}-\log r_{\mathrm{e}}\right) / \sqrt{3},
\end{aligned}
$$

where $\sigma_{0}$ is the central velocity dispersion and $I_{\mathrm{e}} \equiv 10^{-0.4\left(\mathrm{SB}_{\mathrm{e}}-27\right)}$. The solid line shows the observed relation for $V$-band (Pahre 1999), and we could reproduce the observed relations from the $B$-band to the near infrared.

The $\kappa_{1}-\kappa_{2}$ diagram (lower panel) is the face-on view of the fundamental plane. There is no correlation between mass and surface brightness. The $\kappa_{1}-\kappa_{3}$ diagram (upper panel) is the edge-on view. There is a relation with a shallow slope: more massive ellipticals have large 'massto-light ratios'. This 'mass-to-light ratio' is a value defined with $\sigma_{0}$, and is similar not to the total mass-to-light ratio but to the stellar mass-to-light ratio. The origins of the slope are high metallicity, old age, and large baryon fraction for massive ellipticals. Compared with giant ellipticals, dwarf ellipticals are in a different position, having smaller $\kappa_{1}$, smaller $\kappa_{2}$, and larger $\kappa_{3}$, which are consistent with observation (Bender et al. 1992). Dwarf ellipticals have smaller masses and fainter surface brightnesses, and have larger 'mass-to-light ratios' because dwarfs are dark-matter dominated.

Intrinsic scatter exists along the fundamental plane. The origin of scatter is clearly shown with the symbols: merger galaxies (open symbols) have smaller $\kappa_{2}$ and larger $\kappa_{3}$ than non-merger ellipticals (filled symbols). Therefore, the origin of the scatter along the fundamental plane is found to 
be the difference in the merging history. As shown in Figures 12 and 13 of $\mathrm{K} 04$, the dynamical information on the orbits of $N$-body particles is not fully wiped out, even by a major merger, but fairly lost to change the metallicity gradient. In our simulation, the galaxies that undergo the major merger tend to have larger $r_{\mathrm{e}}$ and fainter $I_{\mathrm{e}}$ (see Figure $3 \mathrm{~b}$ and $\mathrm{c}$ ). There is no significant change in $\sigma_{0}$ and total luminosity $L$. From the definition, these result in smaller $\kappa_{2}\left(\propto L \sigma_{0}^{2} r_{\mathrm{e}}^{-5}\right)$ and larger $\kappa_{3}\left(\propto L^{-1} \sigma_{0}^{2} r_{\mathrm{e}}\right)$.

\section{Conclusions and Discussion}

We simulate the chemodynamical evolution of a hundred elliptical galaxies from the CDM initial conditions and succeed in reproducing scaling relations, such as the Faber-Jackson relation and the fundamental plane. At the same time, we reproduce the variety of the internal structures, i.e. no correlation between radial metallicity gradients and galaxy masses.

We should note two problems in the simulation. One is that galactic winds do not occur in large galaxies and star formation never terminates completely. This problem arises from the SPH method and the feedback scheme. If we include the kinetic feedback, surface brightness decreases at the centre, and metal-rich gases blow out. However, these result in much larger effective radii and much shallower metallicity gradients than the observational data (see Figure 14 in K04). The second is that the limited field size of initial conditions causes an artificial cut-off of in the mass accretion and star formation rates. With a wider simulation the star formation continues longer and, thus, colours tend to be too blue. The star formation and feedback schemes need to be modified accordingly. In observed ellipticals, star formation should be truncated at $z \sim 2$ by some process: tidal stripping, effects of active galactic nuclei and so on. This epoch may correspond to the epoch when the galaxy falls into a cluster, where a galaxy moves too fast to undergo mass accretion.

The global properties of elliptical galaxies depend mainly on their masses, while their metallicity gradients are greatly affected by their merging history. Merging histories can thus, in principle, be inferred from the observed metallicity gradients of present-day galaxies. The dispersion in metallicity gradients for galaxies with similar merging histories is not small and so it may be difficult to determine the merging history of an individual galaxy from its metallicity gradient. However, it should be possible to estimate the fractions of non-merger and merger galaxies by using the statistics of metallicity gradients. For example, if the fractions are estimated for field and cluster galaxies, they should provide information concerning environmental effects on galaxy formation.

Available observations for nearby galaxies suggest that non-major-merger galaxies and major-merger galaxies exist in a half-to-half ratio. The observed variation in the metallicity gradients cannot be explained by either monolithic collapse or by major merger alone. Instead, it is reproduced well in the present model, in which both formation processes arise under the CDM scheme.

\section{References}

Bender, R., Burstein, D., \& Faber, S. M. 1992, ApJ, 399, 462

Binggeli, B., Sandage, A., \& Tarenghi, M. 1984, AJ, 89, 64

Carlberg, R. G. 1984, ApJ, 286, 403

Kobayashi, C. 2004, MNRAS, 347, 740 (K04)

Kobayashi, C., \& Arimoto, N. 1999, ApJ, 527, 573

Kobayashi, C., Tsujimoto, T., \& Nomoto, K. 2000, ApJ, 539, 26

Kobayashi, C., Tsujimoto, T., Nomoto, K., Hachisu, I., \& Kato, M. 1998, ApJ, 503, L155

Pahre, M. A. 1999, ApJS, 124, 127

White, S. D. M. 1980, MNRAS, 191, 1p 